\title{
Design Crowdsourcing Supply Chain in Short Life Cycle Products
}

\author{
Dolgion Boldbaatar ${ }^{1}$, and Daeheon Choi $^{1^{*}}$ \\ ${ }^{1}$ College of Business Administration, Kookmin University, 77, Jeongreung-ro, Seongbuk-gu, 02707 \\ Seoul, Korea
}

\begin{abstract}
Design crowdsourcing is the largest open innovation model that can create value with potential consumers. It offers an opportunity to quickly respond to the market by obtaining instant designs from the crowd, freelancing designers with fundamental skills. In addition, it can secure globally innovative competitiveness without financial burden, which is more effective to start-up companies and small and medium enterprises (SMEs) in the field of seasonal product industry. Developing standardized design crowdsourcing supply chain processes and mathematical models is essential to respond to market trends and customer needs in the seasonal product industry. This study has been carried out to determine the best mechanism in the design crowdsourcing supply chain and coordinate each supply chain member whose desires meet each other. Thus, we identify contracts under which conditions can coordinate the crowdsourcing supply chain by a newsvendor model with a manufacturer and a retailer with a crowdsourcing platform. To see that, we study the coordination of the crowdsourcing supply chain through the following contracts: wholesale price, buy-back, and revenue sharing contract. For the forecasting, we present a framework of the design crowdsourcing supply chain and compare supply chain performance under crowdsourcing supply chain coordination. The summarized result shows that the wholesale price contract cannot coordinate the crowdsourcing supply chain efficiently. In contrast, buy-back and revenue sharing contracts can coordinate the crowdsourcing supply chain.
\end{abstract}

Keywords: crowdsourcing, seasonal products, supply contracts, supply chain coordination, newsvendor model

\section{Introduction}

Manufacturing, which requires specialized technology and facility investment, has long been recognized as a large corporation and professional's domain. On the other hand, digital manufacturing such as $3 \mathrm{D}$ printers and $\mathrm{CNC}$ machines are now lowering manufacturing barriers. Now is the time for crowds to design their ideas on computers, share design files, and produce using digital manufacturing [23]. These days with the help of digital manufacturing and production systems, manufacturers can produce products designed by the crowds. Design crowdsourcing is a key action if companies want to continue to be successful.

\footnotetext{
*Corresponding author: dhchoi@kookmin.ac.kr
} 
There are many types of crowdsourcing platforms, but their goals are the same: they connect people and use collective wisdom to tackle innovation challenges. The $3 \mathrm{D}$ printing and digital manufacturing industries have very close neighbors for crowdsourcing platforms. Design crowdsourcing is not just about acquiring new ideas but also improving and fine-tuning ideas. It provides the ability to collaborate with the broader community at a high level [1].

Meanwhile, the life cycle of various goods has been shortening since consumers' lifestyles and trends keep changing at a fast pace. And bringing new products to market with innovation is one of the significant issues in business research today [2]. Recently, in the fashion industry, as customer needs are diversified and supply lead time has been greatly shortened, designs that immediately reflect the latest trends and fast fashion, which compete with rapid product turnover, are in the spotlight. In addition, as competition between companies intensifies, how quickly and efficiently satisfy customer needs is being emphasized as an important success factor in the fashion industry [3]. As the speed of product design development with short life cycle products is getting faster, enterprises need to secure competitiveness through open innovation that can quickly reflect trendy designs [4]. Design crowdsourcing increases the resources available for new product development. In research, managers said that crowdsourcing design as a resource replenishment strategy could simplify and speed up the new product development process [1].

After all, social technologies raise new sensitivities, seeking to breach organizational walls and instill more collaborative mindsets. The multiplication of knowledge channels drove process improvements such as faster time to market and improved product and service quality [5]. Thus, global companies are applying crowdsourcing as a business model of open innovation to product development and production process [6]. Companies in the fast-fashion sector that deal with trendy products with uncertain demand apply a new concept, crowdsourcing supply chain (CSC, Crowdsourcing Supply Chain), to their business models to respond to the market quickly [7]. The crowdsourced design business model refers to how the user becomes the subject of design by opening the product development and design process to the public. It can share and reproduce the created design [6]. In addition, the incremental value of design crowdsourcing is high when the quality of the idea is low. When the initial quality of raw ideas is high, the company can be better with in-house design [1]. Crowdsourcing is the largest open innovation model that can create value by taking a closer look at the opinions and reactions of potential consumers. Also, it is making another form of partnership among companies and consumers.

In the case of SMEs, it is generally challenging to maintain competition without innovation [8]. Crowdsourcing can secure globally innovative competitiveness without financial burden, which is more effective to small and medium enterprises (SMEs). Using the help of Internet users, the company is delegating tasks in which they have no experience. Otherwise, it can cost them a lot of resources [9].

Despite its enormous potential, the crowdsourcing supply chain has yet to be studied theoretically. Although it is being applied to various business fields such as idea-sharing and problem-solving, research on using design crowdsourcing in the supply chain has not been conducted yet. Companies are concerned about the complexity of the crowdsourcing supply chain as it involves the public or consumers to the supply chain online, unlike traditional supply chains. Involving consumers in the production process of new products does not mean that companies lack creativity. What they want is to be closer to consumers, hear their voices, create unique value for them, and replace the traditional way of thinking [10]. In this paper, we intend to suggest a design crowdsourcing supply chain business model that outsources the design of short-term seasonal products whose value decreases after the season passes. In addition, in the process of outsourcing the design from the crowd by the manufacturer, we intend to analyze the impact of design crowdsourcing on the entire supply chain profit. However, when design crowdsourcing is applied to the supply chain, the relational complexity and problems between each entity arise. 
Therefore, we will try to find out which supply chain structure is optimal under design crowdsourcing. We examine crowdsourcing supply chain coordination with the following contracts, wholesale price, buyback, and revenue sharing contract. There are three related research questions addressed in this paper. Firstly, how does design crowdsourcing affect the total supply chain profit? Secondly, which contract can coordinate the design crowdsourcing supply chain? Thirdly, which contracts are worth adopting in it? In the next phases, we perform the numerical analysis to conduct the suggested model.

\section{Literature review}

\subsection{Crowdsourcing}

The term crowdsourcing is a combination of crowd and outsourcing, and entities can access experts faster and lower than traditional channels [24]. The concept of crowdsourcing was introduced in 2006 by Jeff Howe in his article "The Rise of Crowdsourcing" published in the Wired Magazine journal [25]. With the advent of Web 2.0, the mass of people, whose skills are close to experts, increased on platforms, and an economic principle appeared that applied collective intelligence to business [26]. Anyone who can connect to the Internet, whether an expert or an expert, can provide solutions and answers for open outsourcing [9]. Unemployed, enthusiastic publics with overflowing talent and creative imagination generate professionallevel results beyond amateurs [28]. Web 2.0 applications take full advantage of the inherent benefits of the platform: the software is delivered as a continuously updated service, the more people use it, the better it gets, and consumption and remixing come from multiple sources. While providing its data and a form of service, it allows others to mix, create network effects through "participatory architecture." and go beyond the metaphors of Web page 1.0 to provide a rich user experience [11]. Hence, crowdsourcing has the potential to remarkably transform the business processes by incorporating the knowledge and skills that globally dispersed experts, at shorter cycles and low cost [12].

At the heart of every $R \& D$, there is innovation, and the innovation process is conspicuously similar across industries [13]. If the current trend is based on the principle of high prices for a single product, then the crowdsourcing process and consumer personalization provide consumers with an opportunity to obtain a unique product that perfectly meets customers' needs [9]. Several well-known venture companies have used crowdsourcing as their business model, successfully leveraging crowd participation and collaboration, and this business model is rapidly changing the industry [14]. In addition, product ideas with significant improvement needs may benefit from an iterative process, allowing for improvements to ideas driven by crowdsourcing [1]. Innovation strategies moving from closed to open innovation, from $R \& D$ to connection and development, and from competition to cooperation are paving the way for establishing new forms of collaborative production, particularly suitable for SMEs [8]. Therefore, SMEs and global companies can achieve great results by allowing the crowd to perform various activities and functions previously only available to large corporations. Using a crowdsourcing strategy provides companies with essential advantages, such as establishing a closer relationship with consumers and facilitating the optimization of their expectations, without forgetting the potential communication between them [9]. It also allows SMEs to engage in new projects within a complementary approach, allowing them to develop new competencies in the long term [8].

\subsection{Seasonal product}

Recent trends in consumer personalization, diversity, and technological development are accelerating the change from existing products to new products. Due to this change, many 
products are classified as seasonal products. Seasonal products have a low residual value in a market with uncertain demand and have a long manufacturing and procurement period and a short sales period [15]. Rapidly growing electronic products and products in the fashion field, where trends frequently change, rapidly decline in value after a certain period has passed. Even social phenomena are classified seasonally. According to this social phenomenon, the demand of medical device manufacturers and pharmaceutical companies changes with the season.

The seasonal product in this paper is about products that always have to deal with changing trends with innovation and new designs. Also, seasonal products are sold only in the peak season due to factors such as seasons and trends and then form data in the form of a time series that calms down again. As such, it is most important to maintain an appropriate inventory for seasonal products that take the form of a time series that rises and falls due to trend fluctuations and seasonal factors. When calculating the initial supply quantity by intuition, there is a shortage of inventory or bad stock, which leads to promotional activities such as discounts to exhaust the bad stock, hindering profit activities and creating a vicious cycle in the supply chain [17]. Demand for seasonal products has a short peak season throughout the year and changes in trends every year, so past sales information is not very useful [18]. In this paper, seasonal products is categorized where demand plummets after the peak season.

\subsection{Design crowdsourcing}

In design crowdsourcing, any customer and user can also become a designer for a company. Crowdsourcing offers many benefits, especially in the graphic design industry, by providing multiple-choice, creativity, cost, and speedy solutions. The tools used for development, design, and collaboration have undergone a fundamental shift in the past decade. It becomes more powerful and easier to use, even if their prices plummet [22].

Startups selling seasonal products that become unprofitable once a season passes or are out of vogue adopt crowdsourcing for 'designing new products' because they are busy preparing various designs for the next season [8]. As product life cycles are getting shorter, companies need to read customer needs through crowdsourcing quickly. Furthermore, companies are under pressure to produce more innovative products while constrained by internal resources. The needs of modern users are increasingly diversified and advanced because enterprises cannot sufficiently satisfy the needs of these advanced users. It is because enterprises have to pursue profits by producing mass products that satisfy the consumers [23]. On the other hand, upon request, enterprises can easily collaborate with artists and designers to create logos, branding, marketing materials, and more for their business or ideas with design crowdsourcing. When companies know what products or solutions, they want but are looking for executable designs, design crowdsourcing helps make development happen.

The diversity of the crowd makes any activity more attractive. Beyond Prosumers and the Cresumers, the general crowds can now participate in corporate manufacturing activities. The crowd broadens access to a wider variety of competition and ideas and resources than a company can find internally. As the crowd participates from the product planning stage, it is possible to create the maximum effect at the minimum cost by developing products that reflect the needs of consumers but also promoting products from the beginning [19]. Although design requires expertise, many companies outsource freelancers because it is often carried out as a project. The open-minded experts and the system that supports it are essential to realize the public's support rather than a few experts and lead to the right product. Moreover, differentiated print patterns always give fashion manufacturers a competitive advantage in the market [20]. For brands, crowdsourcing is the way of proposing new and original products that will help them organize a brand image to offer unique products, especially in fashion or ready-to-wear products [9]. Companies may be concerned that the open design process will lose control over it, the research interviews show that companies 
can better control the design process while creating slack for their R\&D teams, design through participation in the process, and choosing the right design crowdsourcing platform to iterate with users or designers [1].

There are several companies that utilize design crowdsourcing in their business model. As a representative example of design crowdsourcing, Threadless in the United States is a design-based crowdsourcing platform that makes and sells T-shirts with designs created by the crowd. Anyone can create and upload designs by signing up for the Threadless website. Internet-born design shopping, known as screen-printed graphic T-shirts, was once known (at the time it was thought impossible) as the best example of how to make money on the Internet [29]. The public uploads their designs according to the design guide provided by Threadless. On average, more than 1,000 designs are uploaded a week, and an average of about 50,000 designs are uploaded annually. Selected designers receive $20 \%$ of sales as royalties. The fashion companies generally produced T-shirts' print designs using expensive print patterns from internal experts or external freelancers.

Lego, a Danish toy company, collects ideas through the 'LEGO Ideas' platform launched in 2007 and proceeds to commercialization after user voting and screening. $1 \%$ of sales is provided as a reward to providers whose ideas are adopted on the crowdsourcing platform [28]. This method allows Lego to get unique product designs for free and understand customers' needs. When users try to promote their product ideas and gain likes, they create more buzz around Lego. This, in turn, adds to the mindshare and serves as free promotion for the brand. Lego transforms consumers into active participants to innovate beyond the traditional mass-market model [14]. After collecting creative ideas from the public through their 'LEGO Ideas' platform, they commercialize products with new designs through customer voting and screening processes.

Designhill, a graphic design market based in New Delhi, created infographics as part of its digital media strategy. Rahul Aggarwal founded Designhill with his brothers. As a startup, Aggarwal understands the need for interactive guides, mini-quizzes, and other content. The platform connects clients and designers all over the world. A client who needs a logo, website, and graphic design launches a contest and chooses from designers' work.

Table1. Examples of Design crowdsourcing platforms

\begin{tabular}{|c|c|c|c|c|}
\hline & Description & $\begin{array}{l}\text { Company } \\
\text { (platform) }\end{array}$ & Category & Designer's Revenue \\
\hline \multirow{5}{*}{ Type 1} & \multirow{5}{*}{$\begin{array}{l}\text { The third-party } \\
\text { platform } \\
\text { connects crowds } \\
\text { and clients for } \\
\text { design } \\
\text { crowdsourcing }\end{array}$} & 99 designs & $\begin{array}{l}\text { Logo, Web page, product } \\
\text { package, and other } \\
\text { categories }\end{array}$ & Prize money \\
\hline & & Crowdspring & Custom design, logo & Prize money \\
\hline & & Designcrowd & Logo, app, t-shirts, art, etc. & $\begin{array}{c}\text { Prize money } \\
\text { (minimum \$200) }\end{array}$ \\
\hline & & Fiverr & $\begin{array}{l}\text { Graphic design, Digital } \\
\text { marketing, video, and } \\
\text { animation, etc. }\end{array}$ & $\begin{array}{l}\text { Price is set } \\
\text { by freelancer }\end{array}$ \\
\hline & & Cadcrowd & $\begin{array}{c}\text { 3D design and 3D printing } \\
\text { ideas }\end{array}$ & Prize money \\
\hline
\end{tabular}




\begin{tabular}{|c|c|c|c|c|}
\hline \multirow{4}{*}{ Type 2} & \multirow{4}{*}{$\begin{array}{l}\text { Companies } \\
\text { utilize design } \\
\text { crowdsourcing } \\
\text { and sell } \\
\text { creativity }\end{array}$} & Away to Mars & Clothing designs & $\begin{array}{c}\text { Profit-sharing } \\
(20 \% \text { for the designer) }\end{array}$ \\
\hline & & Threadless & $\begin{array}{l}\text { Screen graphic designed } \\
\text { T-shirts by the crowd }\end{array}$ & $\begin{array}{l}\text { Profit-sharing } \\
\text { (20\% for the designer) }\end{array}$ \\
\hline & & Istockphoto & $\begin{array}{l}\text { High-quality Images and } \\
\text { Illustrations }\end{array}$ & $\begin{array}{c}\text { Approximately } \\
25-45 \text { cents } \\
\text { per image, per month }\end{array}$ \\
\hline & & Spoonflower & $\begin{array}{l}\text { Design crowdsourced } \\
\text { fabric }\end{array}$ & $10 \%$ of the retail price \\
\hline \multirow{3}{*}{ Type 3} & \multirow{3}{*}{$\begin{array}{l}\text { Brands using } \\
\text { crowdsourcing } \\
\text { for open } \\
\text { innovation and } \\
\text { new design }\end{array}$} & LEGO & New Lego product sets & $1 \%$ of sales \\
\hline & & Unilever & New product design ideas & Prize money \\
\hline & & Localmotors & 3D printed car design & Prize money \\
\hline
\end{tabular}

Source: Own Processing.

We classify the chart according to platform types. And Type1 is the crowdsourcing platform in this research work model.

\section{Type 1. The third-party platform connects crowds and clients for design crowdsourcing}

In type1, there are third-party platforms that connect freelance designers to manufacturers. In their platforms, they usually classify freelance designers according to their skills and working experience. They offer cost-effective design solutions, and this kind of platform is popular with small businesses. Access dozens of new design ideas from a diverse pool of talented and capable designers, both professional and amateur, from around the world. Moreover, creative design concepts are available at very affordable prices. So, if companies are looking for a creative logo design, they need to start a contest on the site and enter their design requirements. Soon they will start receiving dozens of design concepts according to their design papers.

\section{Type 2. Companies utilize design crowdsourcing and sell creativity}

Companies that utilize design crowdsourcing and sells creativity are mentioned in type2. The platform companies collect new design ideas from freelance designers. This approach may be more common in start-ups, using and trying collaboration solutions for the first time. Crowds participate in a new designing process without prepayment, then after selection, the designer, whose idea selected, gets certain revenue from profit. As you can see, the freelancer designer's position in this section is higher than the manufacturer and platform owner. It is because organizations that adopt a passive approach to managing crowdsourced IP do not care about crowdsourced IP and are less active in limiting liability. Moreover, the designer has the copyright of product design rights acquired by non-exclusive license [21].

\section{Type 3. Brands using crowdsourcing for open innovation and new design}

Companies using crowdsourcing for open innovation and new design are classified in type 3 . They open projects or contests on their own platform to gather new designs and ideas. Large companies usually facing complex challenges turn to crowdsource to find ideas and possible solutions. This is a collaborative model that prioritizes good ideas and new designs and even rewards freelance 
designers with prize money. They also use crowdsourcing as a marketing tool to reach out to more creative customers. Employees also gain specific experience and knowledge in the narrow areas where the company is concentrated. In contrast, crowds can attract global participants in various companies, territories, and industries that have their interests and motives.

\section{Data and methods - basic order model}

In this paper, we consider the supply chain structure of design crowdsourcing as a two-level supply chain consisting of a supplier and a retailer. An independent platform aimed at crowdsourcing serves as connecting freelancer designers and providers. Because of its characteristics, the products in this research work have a short product life cycle (PLC) and produce small quantity batch production of various types and are sold only for a single period. We assumed that the manufacturer produces multiple items of product on the same production line. The supplier is at the retailer's request in the original design manufacturing (ODM) method in which the supplier takes charge of the design and production. The operation process of the crowdsourcing supply chain proceeds through the following procedures in Figure1.

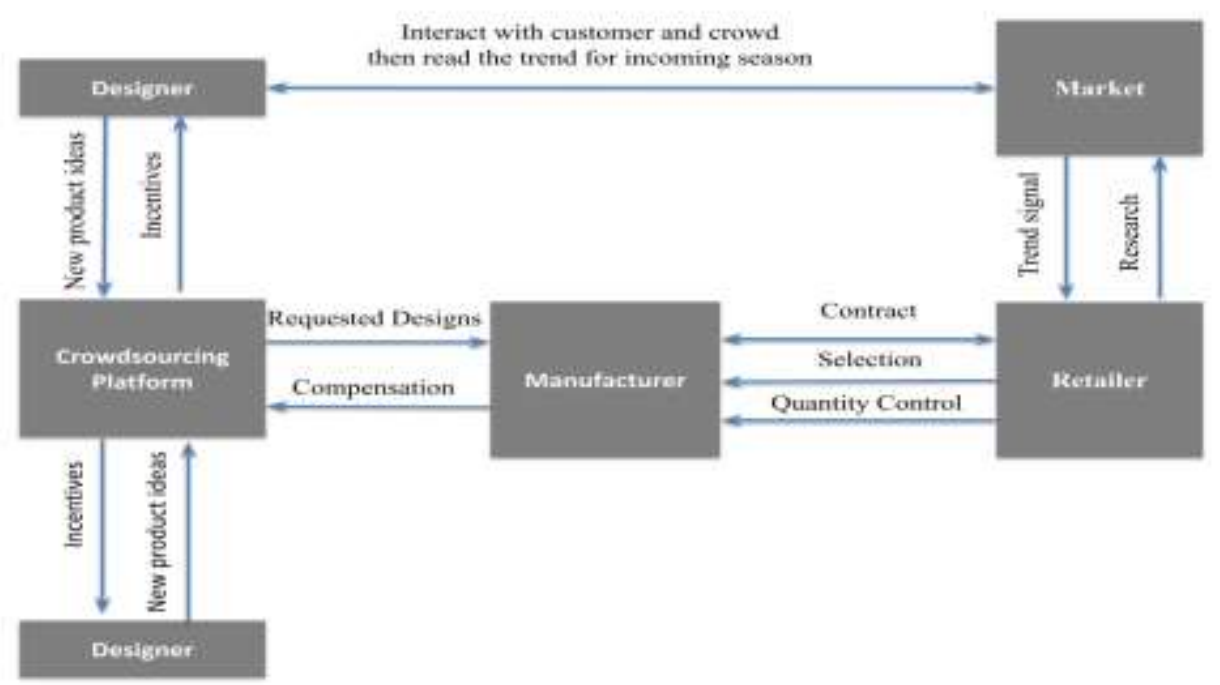

Fig. 1. Design crowdsourcing supply chain process

Source: Own Processing.

\section{Results}

\subsection{Model}

There is one manufacturer and a retailer with a crowdsourcing platform for design in the supply chain. The retailer chooses an order quantity before the start of a single season that has a stochastic demand. Let $D$ be in demand during the selling season. $(D>0)$. Let $F$ be the distribution function of demand and $f$ be its density function: $F$ is differentiable, strictly increasing, and $F(0)=0$. Let $\mu=E[D] . D$ follows the independent and identical distribution. The manufacturer rewards crowdsourced designer $j$ with financial incentives, $r_{j}$, 
if a product designed by designer $j$ in the crowdsourcing platform is selected. The unit production cost, wholesale price, and selling price are denoted by $c_{m}, p_{w}$, and $p$. At the end of the season, any unsold product will be cleared as salvage value $v$ when demand does not exceed sales. Lost sales occur as value $\omega$ when demand exceeds sales. $S(q)$ is expected sales, $\min (q, D)$, expressed by the following.

$$
S(q)=q(1-F(q))+\int_{0}^{q} x f(x) d x=q-\int_{0}^{q} f(x) d x
$$

Let $I(q)$ be the expected leftover inventory and $L(q)$ be the lost sales function. At the end of season, each function for two possible cases can be defined by actual demand occurred during the season, $I(q)=(q-D)^{+}=q-S(q)$ and $L(q)=(D-q)^{+}=\mu-S(q)$. Using these terms, the retailer's profits are:

$$
\pi_{r}(q)=p S(q)-p_{w} q+v I(q)-\omega L(q)
$$

In this case, the retailer's optimal order quantity is:

$$
q_{r}^{*}=F^{-1}\left(\frac{p-p_{w}+\omega}{p-v+\omega}\right)
$$

Meanwhile, crowdsourcing supply chain's profits are:

$$
\pi_{s c}(q)=\left(p-z_{j} r_{j}\right) S(q)-c_{m} q+v I(q)-\omega L(q)
$$

Where $z_{j}=\alpha \tau_{j},\left(\alpha \in(0,1), \tau_{j} \in[0,1]\right) \cdot \tau_{j}$ is a binary variable, taking the value of 1 if a product designed by designer $j$ in the crowdsourcing platform is selected, otherwise, taking the value of 0 . Selected designer $j$ is rewarded at rate $\alpha$ based on sales volume.

Total supply chain's optimal order quantity is:

$$
q_{s c}^{*}=F^{-1}\left(\frac{p-c_{m}+\omega-z_{j} r_{j}}{p-v+\omega-z_{j} r_{j}}\right)
$$

In the next section, we examine the mechanism of crowdsourcing supply chain coordination in the form of several supply contracts with the optimal order quantities of two entities above.

\subsection{Crowdsourcing supply chain coordination}

The following sequence of events occurs in this coordination. The manufacturer provides the contract to the retailer. The retailer accepts or rejects the contract. Assuming the retailer accepts the contract, the retailer submits the order quantity to the manufacturer. The manufacturer produces after selecting the designer's item on the platform. The manufacturer delivers to the retailer before the selling season. Seasonal demand arises. Finally, payments are made between the companies based on the agreed contract. If the retailer rejects the contract, the game will end. 
In design crowdsourcing supply chains, the newsvendor model is not complex, but it is fully to study the coordination mechanism in supply chains. First, which contracts coordinate the design crowdsourcing supply chain? Second, which contracts have sufficient flexibility to allow for any type of the crowdsourcing supply chain's profit? Third, which contracts are worth adopting in it? As a result, contract designers may prefer to propose simple contracts, even if those contracts do not optimize the performance of the supply chain. We examine crowdsourcing supply chain coordination with the following contracts, wholesale price, buyback, and revenue sharing contract. In the next phases, we perform the numerical analysis to conduct the suggested model.

\section{Conclusions}

This paper analyzes the design crowdsourcing supply chain in seasonal products. In the basic order model, we established a design crowdsourcing supply chain with retailer, manufacturer, and design crowdsourcing platforms. Supposing the manufacturer produces multiple items of product on the same production line, and the platform has fundamental designs to pick. In order to coordinate the total members of the supply chain, analyzed contracts; wholesale price, buy-back and revenue sharing contracts. The summarized results are; (1) Wholesale price contract cannot coordinate the crowdsourcing supply chain efficiently. (2) Buy-back contract and revenue sharing contract can coordinate crowdsourcing supply chain as reducing the retailer's loss and hedging the risk under volatile price scheme and fluctuating demand. Using the results, we seek the mechanism to fully coordinate all participants of the design crowdsourcing supply chain, designers, platform, manufacturer, and retailer.

For future research, we discuss several types of future research suggestions. First, it is worth investigating the three-level supply chain in design crowdsourcing including manufacturer, retailer, and the crowds with platforms. This could help the optimization more realistic way when crowds get incentives from retailer. Second, it would be interesting to empirically examine how the design crowdsourcing affects seasonal products' supply chain performance.

\section{References}

1. B. Allen, D. Chandrasekaran, S. Basuroy, Design Crowdsourcing: The Impact on New Product Performance of Sourcing Design Solutions from the "Crowd". Journal of Marketing, 82, 106-123 (2018)

2. J. Hauser, G. J. Tellis, A. Griffin, Research on innovation: A review and agenda for marketing science. Marketing Science, 25(6), 687-717 (2006)

3. H. Park, K. Park, A Study on the Intelligent Quick Response System for Fast Fashion (IQRS-FF). Journal of intelligence and information systems, 16(3), 163-179 (2010)

4. E. Lee, A Study on the Process Innovation of Fast Fashion -Focusing on Developing Management Algorithm of Zara. Journal of the Korean society of design culture, 17(3), 641-651 (2011)

5. M. Harrysson, D. Schoder, A. Tavakoli, The evolution of social technologies. McKinsey Quarterly, 8-12 (2016)

6. H. Park, A Study on Crowdsourcing Design Appearing in Contemporary Fashion Industry. The Research Journal of the Costume Culture, 25(6), 893-912 (2017)

7. J. Li, C. Liu, X. Zeng, N. Zhang, Optimization and Coordination of Crowdsourcing Supply Chain in Fast Fashion Industry. Mathematical Problems in Engineering (2018) 
8. R. Maiolini, R. Naggi, Crowdsourcing and SMEs: Opportunities and Challenges. In: D'Atri A., Ferrara M., George J., Spagnoletti P. (Eds) Information Technology and Innovation Trends in Organizations. Physica-Verlag HD. pp 399-406 (2011)

9. J. Dugout, M. Azouri, J. Decaudin, S. Rochard, Crowdsourcing, Outsourcing to Obtain a Creativity Group. Arab Economic and Business Journal, 8(1/2), 6-15. ISSN 22144625 (2013)

10. A. Bujor, S. Avasilcai, L. Alexa, Co-Creation In Fashion Industry: The Case Of Awaytomars. Annals of the Oradea University: Fascicle Management and Technological Engineering (2017)

11. T. O'Reilly, What Is Web 2.0: Design Patterns and Business Models for the Next Generation of Software, MPRA. Communications \& Strategies, 4578 (2007)

12. M. Vukovic, C. Bartolini, Towards a Research Agenda for Enterprise Crowdsourcing. Leveraging Applications of Formal Methods, Verification, and Validation 425-434 (2010)

13. C. Terwiesch, W. Xu, Innovation Contests, Open Innovation, and Multiagent Problem Solving. Management Science, 54, 1529-1543 (2008)

14. T. Kohler, Crowdsourcing-Based Business Models: How to Create and Capture Value. California Management Review, 57. 63-84 (2015)

15. J. Li, Y. Zhou, W. Huang, Production and procurement strategies for seasonal product supply chain under yield uncertainty with commitment-option contracts. Intern. Journal of Production Economics, 183, 208-222 (2017)

16. S. Noh, S. Gu, S. Jang, A Study on the Improvement of Inventory Management in the Supply Chain Using Seasonal Demand Forecasting. Proceedings of the Spring Joint Conference of the Korean Society of Industrial Engineers, 4179-4205 (2019)

17. H. Park, K. Park, T. Kim, A Computation Model of the Quantity Supplied to Optimize Inventory Costs for the Fast Fashion Industry. Journal of Society of Korea Industrial and Systems Engineering, 35(1), 66-78 (2012)

18. B. Ahn, Sales Forecasting for Inventory Control on Seasonal Fashion Product, Proceedings of the Korean Operations and Management Science Society Conference, 953-959 (2002)

19. J. Park, E.Choi, M. Park, Strategy of Crowdsourcing for Open Innovation. Sejong: Korea Institute for Industrial Economics and Trade (2016)

20. C. Lee, Roles and Possibilities of Collective Intelligence in Fashion Industry - Centered on Crowd Sourcing. Fashion Informationa and Techonlogy, 12(0), 71-79 (2015)

21. J. de Beer, I.P. McCarthy, A. Soliman, E. Treen, Click here to agree: Managing intellectual property when crowdsourcing solutions. Business Horizons, 60(2), 207-217 (2017)

22. K. J. Boudreau, R. L. Karim, Using the Crowd as an innovation Partner. Harvard Business Review [online], Available at: https://hbr.org/2013/04/using-the-crowd-as-aninnovation-partner (2013)

23. N. Kim, J. Yu, Individuals are also emerging as agents of innovation. LG economic research institute [online], Available at: http://www.lgeri.com/report/view.do?idx=18381 (2013)

24. M. K. Pratt, C. Gonsalves, Crowdsourcing [online], Available at: https://searchcio.techtarget.com/definition/crowdsourcing (2006)

25. https://www.wired.com/2006/06/crowds/

26. M. Fashionn, Crowdsourcing, gathers the wisdom of the public 
[online], Available at: https://m.fashionn.com/board/read.php?table=1004\&number=5213 (2011)

27. N. Titus, Threadless: Crowdsourcing Creativity [online], Available at: https://www.racked.com/2017/11/6/16551468/threadless-t-shirts-ecommerce (2018)

28. L. Hyung-Seok, Lego became a hit by introducing the 'crowd source' model, Sisa Journal [online], Available

at: https://www.sisajournal.com/news/articleView.html?idxno=178825 (2018)

29. Racket, What Happened to the Internet's Favorite T-Shirt Company? [online], Available at: https://www.racked.com/2017/11/6/16551468/threadless-t-shirtsecommerce (2017) 